\title{
Intraocular reticulum cell sarcoma: diagnosis by choroidal biopsy
}

\author{
MUZAFFAR H KIRMANI, EDGAR L THOMAS, NARSING A RAO, \\ AND ROBERT P LABORDE*
}

From the Department of Ophthalmology, University of Southern California School of Medicine, and the Estelle Doheny Eye Foundation, Los Angeles, USA

SUMmARY A patient with uveitis and retinal pigment epithelial detachments underwent an extensive medical examination and pars plana vitrectomy in an attempt to confirm the clinical diagnosis of reticulum cell sarcoma. None of these examinations revealed the presence of malignancy. Transscleral biopsy of subretinal lesions confirmed the histopathological diagnosis of reticulum cell sarcoma. The patient was begun on systemic chemotherapy and external beam radiation to the eye and orbit, with resultant preservation of the eye, vision, and probable extension of life because of early detection of reticulum cell sarcoma by choroidal biopsy. This technique may be advantageous in the diagnosis cases of eyes with clinical evidence of reticulum cell sarcoma, no systemic signs of malignancy, and negative vitreous biopsies.

Reticulum cell sarcoma is a highly malignant multicentric form of non-Hodgkin's lymphoma that may primarily affect the eye and central nervous system. Intraocular involvement typically presents as a picture of unilateral or bilateral chronic posterior uveitis that is poorly responsive to systemic corticosteroid or antimetabolite therapy.

Gass and colleagues' have described the presence of multifocal solid pigment epithelial detachments to be virtually pathognomonic for reticulum cell sarcoma. Ocular findings may precede central nervous system involvement by months or even several years. Patients with reticulum cell sarcoma are typically 40 years of age or older. Confirmation of the diagnosis of ocular reticulum cell sarcoma has traditionally been based on histopathological study of post-mortem eyes, enucleated eyes, or vitreous specimens. Michels and colleagues ${ }^{2}$ reported two cases in which the diagnosis of reticulum cell sarcoma was made on vitreous aspirate obtained by pars plana vitrectomy. Peyman and colleagues ${ }^{3}$ reported a case in which the diagnosis was made by full thickness eye wall biopsy.

Establishing the diagnosis of intraocular reticulum

Correspondence to Edgar L Thomas, MD, Estelle Doheny Eye Foundation, USC School of Medicine, 1355 San Pablo Strect, Los Angeles, CA 90033, USA.

* Department of Ophthalmology/LSU Eyc Center, Louisiana State University Medical Center, New Orleans, LA, USA. cell sarcoma is important with respect to treatment of ocular complications and recognition of systemic disease. Since the central nervous system is commonly affected by this tumour, computerised tomography and other diagnostic methods are indicated. Early diagnosis is important, because both ocular and central nervous system reticulum cell sarcomas are highly sensitive to irradiation, which may preserve visual function and prolong life.

Recent reports have emphasised the importance of vitreous biopsy in diagnosing chronic cellular infiltration of the vitreous in reticulum cell sarcoma. ${ }^{24} \mathrm{We}$ here report a case in which the vitreous biopsy was negative, and the diagnosis of ocular reticulum cell sarcoma was made by transscleral choroidal biopsy.

\section{Case report}

A 67-year-old woman presented with a one-year history of vitreous floaters and decreased vision involving the right eye. In April 1985 she was treated with cryopexy for a retinal tear in the right eye. No other ocular abnormality was noted at that time. Two weeks after cryopexy, vision in the right eye decreased further. Examination at this time revealed elevated retinal lesions in the posterior pole and visual acuity of 20/100 in the right eye. A presumptive diagnosis of chorioretinitis was made. Clindamycin therapy was begun and a single corticosteroid injec- 


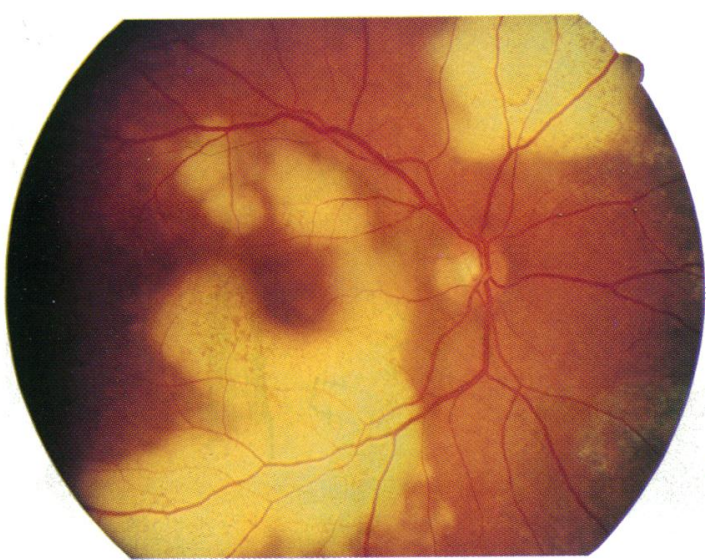

Fig. 1 Fundus photograph of the right eye showing multiple subretinal lesions in the posterior pole.

tion was given transconjunctivally beneath Tenon's capsule. After this therapy the vision improved to $20 / 60$, but two weeks later additional large posterior lesions, as well as many satellite subretinal lesions, were noted in the posterior pole (Figs. 1,2).

An investigation for reticulum cell sarcoma was performed. General physical examination, neurological testing, breast examination, computerised tomography of the head, and chest $x$-ray were normal. The white cell count, blood chemistry, antinuclear antibody test, erythrocyte sedimentation rate, fluoresceinated treponemal antibodies, toxoplasmosis titre, cytomegalic virus titre, and urine analysis were all within normal limits; a purified protein derivative skin test was negative.

The patient's past medical history was significant for hypertension, for which she was being treated with prazosin hydrochloride (Minipress) $1 \mathrm{mg}$ three times a day. She had a history of hysterectomy with

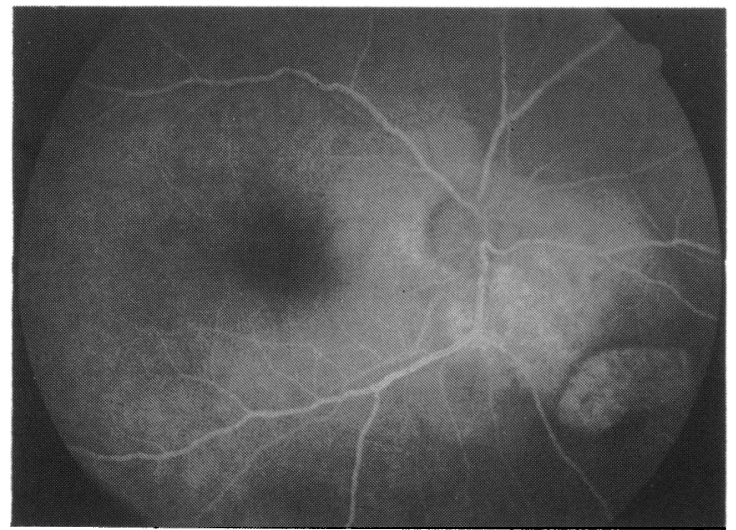

Fig. 3 Arteriovenous phase of the fluorescein angiogram of the right eye showing early blockage of background choroidal vasculature.

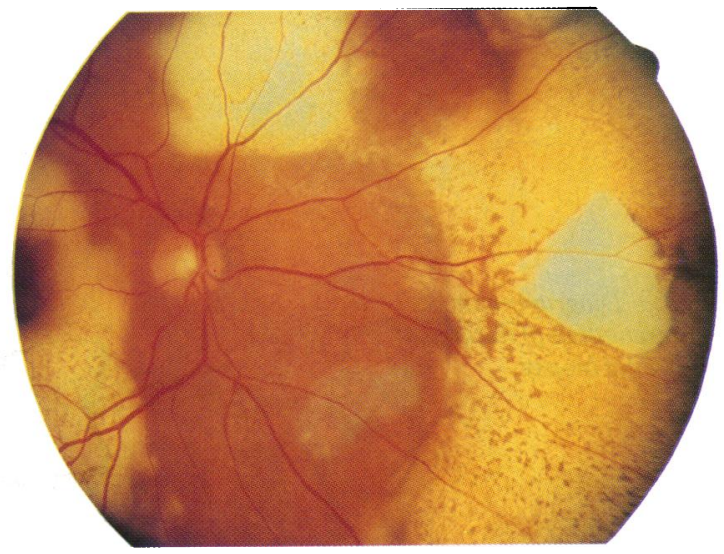

Fig. 2 Fundus photograph of the right eye showing lesions in the nasal periphery.

bilateral salpingo-oophorectomy for benign fibroids of the uterus and was taking oestrogen (Premarin) $0.625 \mathrm{mg}$ five days per week for menopausal symptoms. Her family history included one brother with hypernephroma.

The patient underwent a diagnostic pars plana vitrectomy of the right eye on 10 June 1985 . Cytological preparations revealed a non-specific chronic inflammatory cell infiltration without obvious malignant cells. Ocular examination four days later on referral to the Estelle Doheny Eye Clinic/University of Southern California Department of Ophthalmology showed best corrected visual acuity of 20/100 in the right eye and 20/50 in the left eye; vision in the left eye improved to $20 / 40$ with pinhole. The pupils were $4 \mathrm{~mm}$ on both sides and were equally reactive to light; there was no afferent pupillary defect. The results of external examination of the lids, lacrimal glands and lymph nodes were within normal limits.

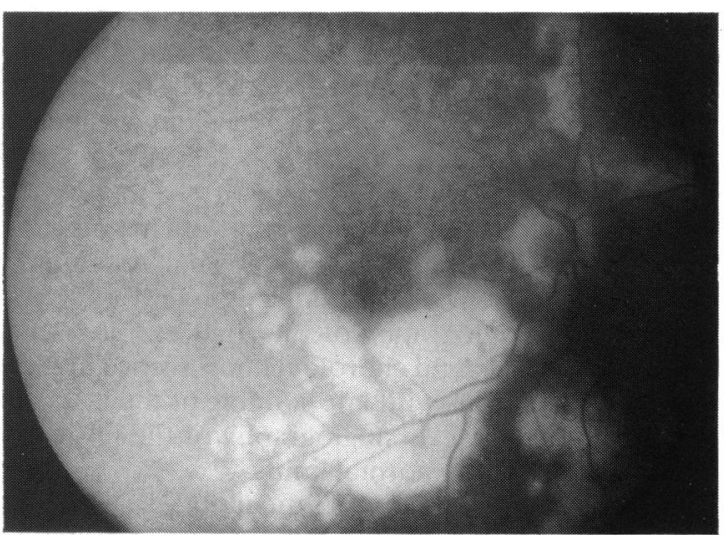

Fig. 4 Fluorescein angiogram of right eye showing late staining of the subretinal lesions. 
Fig. 5 Diagrammatic representation of the area of choroidal biopsy.
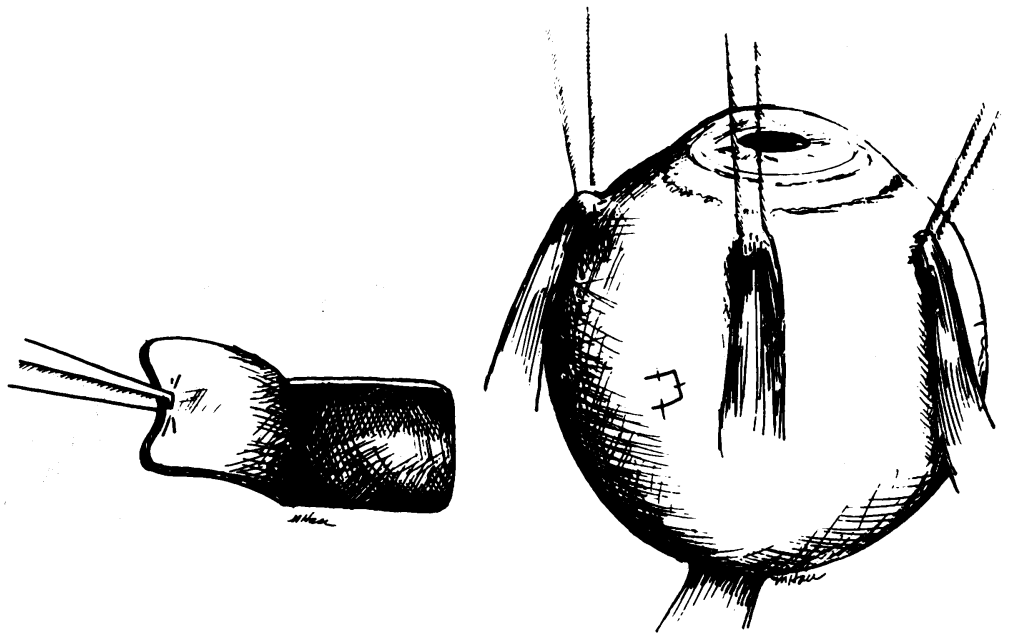

Fine endothelial keratic precipitates with trace flare in the anterior chamber were observed on slit-lamp examination of the right eye. There was no evidence of iris nodules. The lens showed mild nuclear sclerosis, and the vitreous cavity contained a moderate number of cells. Slit-lamp examination of the left eye showed trace cells and flare in the anterior chamber and mild cells in the vitreous cavity.

Funduscopic examination of the dilated right eye disclosed several raised, lobulated, yellowish, subretinal lesions involving the posterior pole and equatorial region; the peripheral retina appeared normal. Several of these subretinal lesions were interconnected by pigment clumping on their surfaces. Many small satellite lesions were noted. Submacular and perimacular lesions beneath the retinal pigment epithelium were also present. The optic nerve was unremarkable. Fundus examination of the dilated.left eye was unremarkable except for a few yellowish drusen-like deposits in the temporal retinal periphery. Ultrasonography of the right eye showed diffuse, highly reflective echoes in the choroid, with shallow serous elevation of the retina and/or pigment epithelium. There was mild to highly reflective thickening of the choroid in the left eye. Ultrasonographic findings were thought to be consistent with metastatic tumours of the choroid. However, ocular reticulum cell sarcoma could not be excluded as a diagnosis.

Fluorescein angiography of the right eye showed diminished background choroidal fluorescence in the vicinity of the lesions with late staining of the lesions (Figs. 3, 4). Fluorescein angiography of the left eye was essentially within normal limits.

The clinical impression was reticulum cell sarcoma involving predominantly the right eye or metastatic carcinoma from either the breast or lung. Repeat physical examination, however, including bone marrow biopsy, lumbar puncture, computerised tomography of the head, complete blood count, blood chemistry, and liver function tests did not reveal evidence of lymphoma or primary carcinoma. In a further attempt to establish a definitive diagnosis the patient underwent a transscleral choroidal biopsy on 25 July 1985 .

Choroidal biopsy was performed under local anaesthesia. The area to be biopsied was marked on the sclera by scleral indentation using indirect ophthalmoscopy. A $2 \times 2 \mathrm{~mm}$ full-thickness scleral window was dissected (Figs. 5, 6) and an excisional choroidal biopsy obtained by lifting the choroid through the window with forceps and cutting it free. Clear liquid vitreous fluid drained from the biopsy site in this previously vitrectomised eye. The scleral window was sutured with 9-0 nylon sutures. The vitreous cavity was immediately filled with a $20 \%$ sulphur hexafluoride gas/air mixture. Cryopexy was applied to the area of biopsy under indirect ophthalmoscopic control. The retina has remained attached for eight months postoperatively. There have been no apparent complications from this biopsy procedure.

Biopsy yielded four friable fragments of tissue, each measuring approximately $1 \mathrm{~mm}$ in greatest dimension. The tissue was fixed in half strength Karnovsky's solution and embedded in paraffin. Sections were cut and stained with either haematoxylin and eosin or periodic acid Schiff reagent. Microscopic examination revealed large round cells with hyperchromatic nuclei and sparse cytoplasm. Admixed with these abnormal subpigment epithelial cells were numerous necrotic cells. The choroid showed diffuse lymphocytic infiltration without the subretinal pigment epithelial space was consistent with reticulum cell sarcoma (Figs. 6, 7). abnormal cells. Cytologically the cellular infiltrate in 


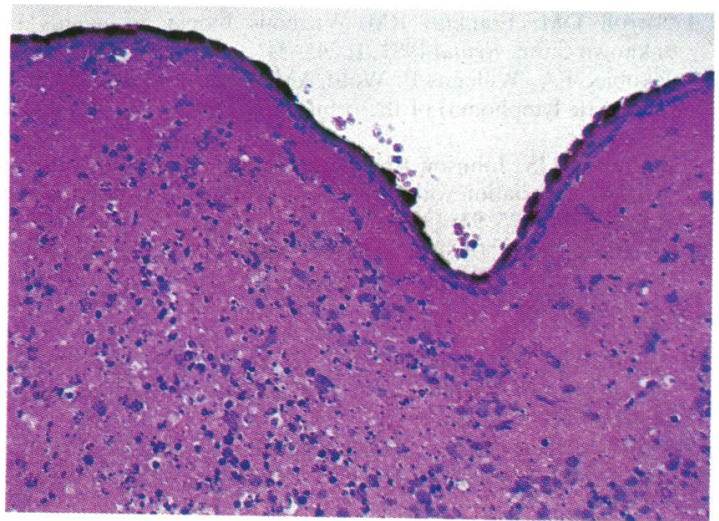

Fig. 6 Subretinal pigment epithelial infiltration by malignant cells. Periodic acid Schiff.

The patient was begun on external beam radiation to the posterior segments and orbits bilaterally. Whole brain and orbital (choroidal) irradiation of 4000 rad was delivered in 180-rad fractions. Parallelopposed ports of cobalt- 60 down to the level of the second cervical vertebra were used. Twenty-two fractions were given over 33 days. Methotrexate was given intrathecally via lumbar puncture in $12 \mathrm{mg}$ doses at weekly intervals for one month for a total of $48 \mathrm{mg}$. Leucovorin, $25 \mathrm{mg}$, in six divided doses over 36 hours was given midway through the methotrexate therapy. Within two weeks the best corrected visual acuity had improved to $20 / 60$ in the right eye with partial resolution of the submacular lesions. At eight months the best corrected vision was 20/40 on the right with no evidence of recurrence of the ocular lesions (Fig. 8) and no apparent complications of the choroidal biopsy procedure.

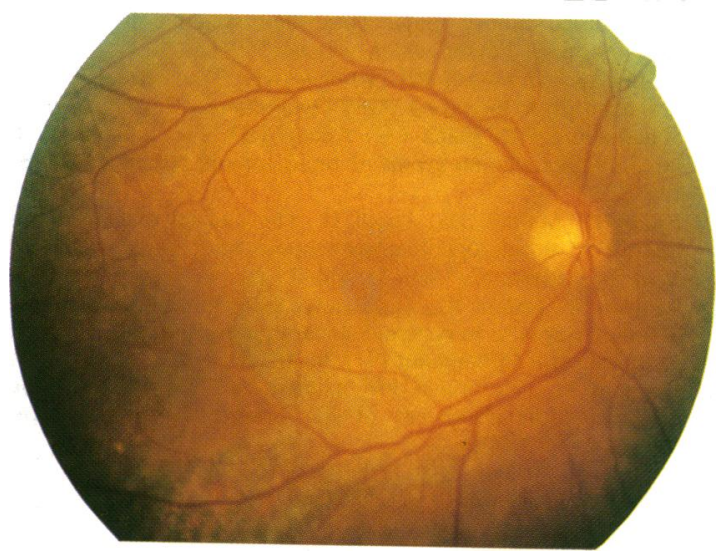

Fig. 8 Fundus photograph eight months after biopsy. Mild mottling of the retinal pigment epithelium is present in areas of previous tumefaction.

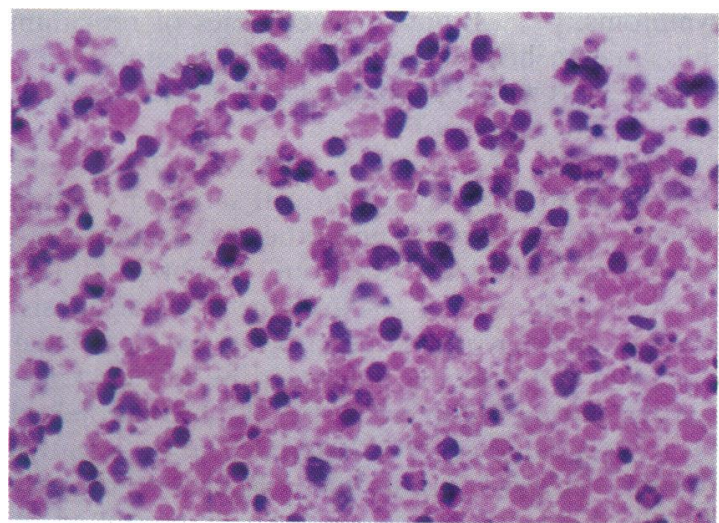

Fig. 7 Biopsy specimen showing malignant cells with scant cytoplasm and large hyperchromatic nuclei. Periodic acid Schiff.

\section{Discussion}

Ocular reticulum cell sarcoma, whether primary or secondary, is an uncommon but highly malignant form of non-Hodgkin's lymphoma. Numerous cases of orbital and intraocular involvement have been

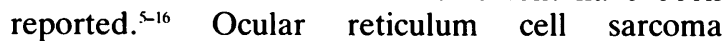
commonly occurs in synchrony with central nervous system involvement. ${ }^{17}$ Barr and colleagues ${ }^{12}$ reviewed 14 cases of intraocular reticulum cell sarcoma, none of which subsequently showed systemic malignancy; Klingele and Hogan ${ }^{13}$ reported eight cases, of which only one later developed systemic disease. Similar discrepancies in nature and incidence of dissemination are found in other case reports. ${ }^{121+15}$ Most authorities consider ocular reticulum cell sarcoma to represent a manifestation of multicentric reticuloendothelial malignancy. Ocular findings are often among the first to become clinically evident.

Classically, patients with ocular reticulum cell sarcoma present with long standing panuveitis, anterior chamber reaction, decreased visual acuity, vitreous cellular reaction, and refractoriness to therapy. Increased intraocular pressure, keratic precipitates, chorioretinal infiltrates, and serous retinal detachments may also be present. While most cases of intraocular reticulum cell sarcoma affect the posterior segment of the globe, involvement of the iris and ciliary body has also been reported. Vitreous cellular reactions have been described as sometimes resembling primary inflammation, thereby failing to show evidence of intraocular malignancy. ${ }^{18}$ Because the central nervous system is frequently affected, computerised tomographic scans and careful neurological examination are strongly recommended. It has been suggested that reticulum cell sarcoma must always be kept in mind whenever patients with chronic idiopathic uveitis present with neurological 
symptoms. ${ }^{169^{1013}}$ Biopsy proved cases of reticulum cell sarcoma histopathologically show large hyperchromic cells with sparse cytoplasm, indented nuclei, and prominent nucleoli.

Cytological confirmation of intraocular lesions suspected of being reticulum cell sarcoma is often difficult. Michels et al. ${ }^{2}$ and others ${ }^{4}$ have described the use of pars plana vitrectomy to obtain filtered vitreous aspirates in establishing the diagnosis. Peyman and coworkers ${ }^{3}$ described a case of ocular reticulum cell sarcoma diagnosed by full thickness eye wall biopsy. Brain biopsy has also been proposed as a potential diagnostic approach after negative vitreous biopsy. ${ }^{6}$ Lang and associates ${ }^{7}$ recently described an interesting case which presented as bilateral panuveitis with multifocal, discrete retinal lesions. Pars plana vitrectomy failed to confirm the suspected diagnosis of ocular reticulum cell sarcoma. Three years later, at necropsy, examination revealed reticulum cell sarcoma involving the brain and both eyes.

With proper technique it is possible to obtain a biopsy specimen of retina and choroid without significant complications. ${ }^{1421}$ Potential complications of choroidal biopsy include: intraocular haemorrhage, retinal detachment, choroidal detachment, endophthalmitis, and retinal incarceration into the biopsy site. Transscleral choroidal biopsy offers a promising alternative diagnostic approach to lesions that are clinically suggestive of reticulum cell sarcoma but have yielded negative vitreous biopsies. Because these lesions are often responsive to irradiation and chemotherapy, early diagnosis with prompt therapeutic intervention may contribute to the preservation of visual function and prolongation of life. ${ }^{22-25} \mathrm{We}$ believe that transscleral biopsy is indicated in patients with negative vitreous biopsy and strong clinical evidence of this life-threatening malignancy.

This work was supported by NEI Grant EYO-3040.

\section{References}

1 Gass JDM, Sever RJ, Clarkson JG, Blumenkranz M, Wind CA, Shugarman R. Multifocal pigment epithelial detachments by reticulum cell sarcoma: a characteristic funduscopic picture. Retina 1984; 4: 135-43.

2 Michels RG, Knox DL, Erozan YS, Green WR. Intraocular reticulum cell sarcoma: diagnosis by pars plana vitrectomy. Arch Ophthalmol 1975; 93: 1331-5.

3 Peyman GA, Juarez CP, Raichand M. Full-thickness eye-wall biopsy: long-term results in 9 patients. BrJ Ophthalmol 1981; 65: 723-6.
4 Carroll DM, Franklin RM. Vitreous biopsy in uveitis of unknown cause. Retina 1981 ; 1 : 245-51.

5 Jakobiec FA, Williams P, Wolff AM. Reticulum cell sarcoma (histocytic lymphoma) of the orbit. Surv Ophthalmol 1978; 22: 255-70.

6 Kennerdell JS, Johnson BL, Wisotzkey HM. Vitreous cellular reaction: association with reticulum cell sarcoma of brain. Arch Ophthalmol 1975; 93: 1341-5.

7 Lang GK, Surer JL, Green WR, Finkelstein D, Michels RG, Maumenee AE. Ocular reticulum cell sarcoma: clinicopathologic correlation of a case with multifocal lesions. Retina 1985; 5: 79-86.

8 Neault RW, Van Skoy RE, Okazaki H, MacCarty CS. Uveitis associated with isolated reticulum cell sarcoma of the brain. Am J Ophthalmol 1972; 73: 431-6.

9 Minckler DS, Font RL, Zimmerman LE. Uveitis and reticulum cell sarcoma of brain with bilateral neoplastic seeding of vitreous without retinal or uveal involvement. Am J Ophthalmol 1975; 80: 433-9.

10 Rosenbaum TJ, MacCarty CS, Buettner H. Uveitis and cerebral reticulum-cell sarcoma (large-cell lymphoma): case report. J Neurosurg 1979; 50: 660-4.

11 Appen RE. Posterior uveitis and primary cerebral reticulum cell sarcoma. Arch Ophthalmol 1975; 93: 123-4.

12 Barr CC, Green WR, Payne JW, Knox DL, Jensen AD, Thompson RL. Intraocular reticulum cell sarcoma: clinicopathologic study of four cases and review of the literature. Surv Ophthalmol 1975; 19: 224-39.

13 Klingele TG, Hogan MJ. Ocular reticulum cell sarcoma. Am J Ophthalmol 1975; 79: 39-47.

14 Vogel MH, Font RL, Zimmerman LE, Levine RA. Reticulum cell sarcoma of the retina and uvea: report of six cases and review of the literature. Am J Ophthalmol 1968; 66: 205-15.

15 Kim EW, Zakov ZN, Albert DM, Smith TR, Craft JL. Intraocular reticulum cell sarcoma: a case report and literature review. Graefes Arch Klin Exp Ophthalmol 1979; 209: 167-78.

16 Char DH, Margolis L, Newman AB. Ocular reticulum cell sarcoma. Am J Ophthalmol 1981; 91: 480-3.

17 Kattach JC, Jenkins RB, Pilkerton AR, Rao NA, Manz HJ, Frazer M. Multifocal primary ocular and central nervous system malignant lymphoma. Ann Ophthalmol 1981; 13: 589-93.

18 Shields JA. Intraocular lymphoid tumors and leukemias. Diagnosis and management of intraocular tumors. St Louis: Mosby, 1983: 634-40.

19 Constable IJ, Slatter DH, Horne R. Chorioretinal biopsy in dogs. Invest Ophthalmol Vis Sci 1980; 19: 603-9.

20 Constable IJ, Chester GH, Horne R, Harriott JF. Human chorioretinal biopsy under controlled systemic hypotensive anaesthesia. Br J Ophthalmol 1980; 64: 559-64.

21 Griffin JR, Straatsma BR, Kreiger AE. Transvitreal chorioretinal biopsy in the rabbit. Am J Ophthalmol 1975; 79: 25-8.

22 Schaumburg HH, Plank CR, Adams RD. The reticulum cell sarcoma-microglioma group of brain tumors. Brain 1972; 95: 199-212.

23 Fisher D, Mantell BS, Urich $H$. The clinical diagnosis and treatment of microgliomatosis: report of a case. J Neurol Neurosurg Psychiatry 1969; 32: 474-8.

24 Sullivan SF, Dallow RL. Intraocular reticulum cell sarcoma: its dramatic response to systemic chemotherapy and its angiogenic potential. Ann Ophthalmol 1977; 9: 401-6.

25 Burstein SD, Kernohan JW, Uihlein A. Neoplasms of the reticuloendothelial system of the brain. Cancer 1963; 16: 289-305.

Accepted for publication 10 October 1986. 